\section{c-MET-Inhibitor bei Lebertumoren aktiv}

Für das nicht operable hepatozelluläre Karzinom (HCC) ist als einzige systemische Therapie der BRAF-Inhibitor Sorafenib zugelassen. Bei einer Tumorprogression nach Primärtherapie gibt es derzeit keine weitere Option Ein neuer therapeutischer Ansatz betrifft den Rezeptor des HepatozytenWachstumsfaktors (HGF) CMET, der eine bedeutende Rolle bei der Proliferation und Metastasierung von Tumorzellen spielt und beim HCC häufig überexprimiert ist. Nach viel versprechenden Phase-I-Daten wurden Wirksamkeit und Sicherheit von Tivantinib, einem selektiven cMET-Inhibitor, in einer randomisierten placebokontrollierten Phase-II-Studie mit 107 Patienten untersucht [Rimassa L et al. J Clin Oncol. 2012; 30 (suppl; abstr 4006)]. Die Therapie mit Tivantinib verlängerte die Zeit bis zur Krankheitsprogression, dem primären Endpunkt der Studie, mit median 6,9 Wochen gegenüber 6,0 unter Placebo zwar signifikant, aber eher bescheiden (HR,64; $p=0,04$ ), erklärte Lorenza Rimassa, Rozzano, Italien. Beim Gesamtüberleben war kein Unterschied festzustellen. Stratifizierte man die Patienten jedoch nach der Höhe der cMet-Expression, verdoppelte sich die mediane Zeit bis zur Progression bei den Patienten, deren Tumoren eine hohe Expression von c-MET aufwiesen, gegenüber Placebo (11,7 vs. 6,1 Wochen; HR 0,43; $p=0,03)$. Auch das Gesamtüberleben war trotz Crossovers eines Teils der Patienten signifikant besser als bei den Patienten, die Placebo erhalten hatten (7,2 vs. 3,8 Monate; HR 0,38; $p=0,01)$. Bei niedriger oder fehlender c-MET-Expression war hingegen kein Nutzen des Inhibitors zu erkennen. Abgesehen von Zytopenien, die unter Tivantinib häufiger auftraten, war die Toxizität in beiden Armen vergleichbar. Damit zeichne sich ein Weg für eine individualisierte Therapie mit Tivantinib bei HCC-Patienten mit hoher cMET-Expression ab, resümierte Rimassa. Eine Phase-III-Studie werde derzeit geplant.

Josef Gulden

\section{Reduktion von Fatique-Symptomen durch amerikanischen Ginseng} Einen phytotherapeutischen Ansatz zur Supportivtherapie stellte Debra L Barton aus Rochester, NY/USA, vor. Zusammen mit ihren Kollegen untersuchte sie in einer Phase-III-Studie die Wirksamkeit von amerkanischem Ginseng (Panax quniquefolius) im Hinblick auf die Fatique-Symptome [Barton DL et al. J Clin Oncol. 2012;30(suppl:abstr. 9001)]. Insgesamt 364 Patienten, davon mehr als die Hälfte an Brustkrebs erkrankt, erhielten randomisiert entweder das Ginseng-Präparat oder Placebo. Bereits nach vier Wochen zeigte sich die Wirksamkeit der Ginseng-Therapie anhand des MFSI (Multidimensional Fatique Symptom Inventory), der mit 14,4 deutlich höher war als im PlaceboArm mit 8,2. Nach acht Wochen war der Unterschied weiter gewachsen und signifikant mit 20,0 versus 10,3 ( $p=0,003)$. Nebenwirkungen, darunter Übelkeit und Erbrechen, waren moderat und in beiden Gruppen vergleichbar häufig. Interessant war, dass besonders die Patienten profitierten, die ihre Krebsbehandlung noch nicht abgeschlossen hatten.

Doris Berger
Vitamin D gegen Muskel- und Gelenkschmerzen und Fatique Viele postmenopausale Patientinnen mit hormonrezeptorpositivem Brustkrebs, entwickeln unter einer adjuvanten Therapie mit einem Aromatasehemmer (Al) muskuloskelettale Komplikationen und Fatique-Symptome. Nicht selten brechen sie deswegen die Therapie ab. Weil viele Brustkrebspatientinnen einen Vitamin-D-Mangel aufweisen, untersuchten Qamar J. Khan aus Kansas City, MO/USA, und Kollegen, ob eine Vitamin-DSupplementierung diese Nebenwirkungen mindern und möglicherweise damit auch die Compliance und das Behandlungsergebnis verbessern kann [Khan QJ et al. J Clin Oncol. 2012;30(suppl:abstr.9000)]. Insgesamt 180 Patientinnen erhielten eine Standard-Al-Therapie mit Letrozol plus 600 IU Vitamin $D_{3}$ und 1.200 mg Kalzium täglich sowie zusätzlich randomisiert entweder weitere 30.000 IU Vitamin $\mathrm{D}_{3}$ per os pro Woche oder Placebo. Die Daten von 147 der 160 in die Studie aufgenommen Patienten konnten ausgewertet werden. Von den Patientinnen, die zusätzlich das hochdosierte Vitamin $\mathrm{D}_{3}$ erhalten hatten, litten nach 24 Wochen signifikant weniger unter muskuloskelettalen Schmerzen als diejenigen der Placebogruppe (38 versus $61 \%, p=0,008)$. Auch Fatique-Symptome besserten sich unter der VitaminD-Supplementierung im Vergleich zum Kontrollarm. Die zusätzliche Gabe von hochdosiertem Vitamin D zur adjuvanten Aromatase-Inhibition (Al) mit Letrozol sei sicher, reduziere die Al-bedingten muskuloskelettalen Komplikationen, verbessere die Lebensqualität der Patientinnen und könne so möglicherweise dazu beitragen, die Compliance zu verbessern, folgerte Khan.

Doris Berger

NSCLC: Irreversible ErbB-Hemmung erfolgreich

Die bisher verfügbaren Inhibitoren von Rezeptoren der ErbB-Familie (im wesentlichen EGFR und HER2) binden reversibel an ihre Zielstruktur. Jetzt wurde erstmals die Strategie einer irreversiblen Hemmung dieser Rezeptoren eingeschlagen. Der irreversible Inhibitor Afatinib ist oral verfügbar und wurde kürzlich in der Phase-III-Studie LUX-Lung 3 bei Patienten mit unbehandelten, fortgeschrittenen Adenokarzinomen der Lunge und aktivierenden Mutationen des EGFR gegen die Kombination Cisplatin plus Pemetrexed getestet [Yang JC-H et al. J Clin Oncol. 2012;30(suppl;abstr LBA7500)] . Wie James Chih-Hsin Yang aus Taipei, Taiwan, berichtete, war Afatinib nicht nur bezüglich der Ansprechrate mit 56,1 versus $22,6 \%$, sondern auch beim PFS mit im Median 11,1 gegenüber 6,9 Monaten deutlich überlegen ( $H R$ 0,58; $p=0,0004$ ). Die Wirksamkeit konnte in allen untersuchten Subgruppen festgestellt werden. Grad-3- und -4-Nebenwirkungen waren in beiden Armen etwa gleich häufig, aber die Verschlechterung tumorbedingter Symptome wie Husten, Dyspnoe und Schmerzen wurde durch Afatinib durchweg länger aufgehalten als durch die Chemotherapie. Auch die Lebensqualitätsdaten sprechen laut Yang für den Inhibitor.

Josef Gulden
Auch sie lebten signifikant länger progressionsfrei unter der Dabrafenib-Therapie (HR 0,32 95\%; KI 0,17-0,60). Die radiologisch gesicherte Ansprechrate lag unter Dabrafenib bei $50 \%$, in der DTIC-Gruppe jedoch nur bei $6 \%$. Die Nebenwirkungen waren erwartungsgemäß unterschiedlich, aber beherrschbar - in beiden Armen brachen nur $3 \%$ der Patienten aufgrund von schwerwiegenden Symptomen die Therapie ab. Allerdings wurden laut Hauschild unter der Dabrafenib-Therapie neue Basalzellkarzinome und primäre Melanome beobachtet.

\section{Auch bei Hirnmetastasen hochwirksam}

Diese guten Ergebnisse bestätigte eine Phase-II-Studie, in der darüber hinaus ein besonderer Fokus auf Hirnmetastasen gelegt wurde. Etwa ein Viertel aller Patienten mit fortgeschrittenem metastasiertem Melanom Stadium IV leiden bereits zum Zeitpunkt der Diagnosestellung unter Hirnmetastasen. Von ei- ner beispiellosen Ansprechrate und einem sichtlichen Überlebensvorteil sprach John M Kirkwood, Pittsburg, PA/USA, in Bezug auf die Wirksamkeit von Dabrafinib bei diesen Patienten. Er stellte in Chicago die Ergebnisse einer offenen Phase-II Studie vor, in der 172 Patienten, mit einet BRAFV600E- oder BRAFV600k-Mutation aufwiesen, mit $150 \mathrm{mg}$ Dabrafenib behandelt worden waren [Kirkwood JM et al. J Clin Oncol. 2012; 30(suppl;LBA8501)]. Mit intrakraniellen Ansprechraten von 39 bzw. $31 \%$ (ohne bzw. mit Vorbehandlung der Hirnmetastasen) und einer intrakraniellen Krankheitskontrolle bei mehr als $80 \%$ der Patienten geht Kirkwood davon aus, dass gerade bei Patienten mit Hirnmetastasen künftig häufiger eine systemische Therapie mit Dabrafenib erwogen werden sollte. Doris Berger

Bericht von der Jahrestagung der American Society of Clinical Oncology (ASCO) vom 1.-5. Juni 2012 in Chicago IL/USA 